\title{
DETERMINACIÓN DEL TIPO DE ANEMIA Y SU RELACIÓN CON LA INGESTIÓN ALIMENTARIA Y MARCADORES BIOQUÍMICOS EN PACIENTES CON CÁNCER CÉRVICO UTERINO
}

\author{
DETERMINATION OF THE TYPE OF ANEMIA AND ITS \\ RELATIONSHIP TO FOOD CONSUMPTION AND BIOCHEMICAL \\ MARKERS IN PATIENTS WITH CERVICAL CANCER
}

\author{
Frida E. Bichara (1), Octavio Amancio (2), Gabriela Gutiérrez-Salmeán (2), Vanessa Fuchs (2) \\ (1) Universidad Anáhuac Norte \\ (2) Servicio de Oncología, Hospital General de México, México.
}

\begin{abstract}
Introduction: Anemia is a syndrome frequently present in cancer patients and it affects their quality of life. In cervical cancer, anemia develops in most of the patients, without knowledge of its possible cause: transvaginal bleeding presents in advanced stages, or a diet low in iron and other micronutrients can also contribute to this phenomenon. Objective: To determine the type of anemia, serum levels of iron, ferritin, transferrine, folic acid and vitamin $B_{12}$ and relate them with dietary intake. Material and methods: We conducted a transversal study in 55 cervical cancer patients without neither active transvaginal bleeding nor oncologic treatment. A blood sample was taken in order to determine the biochemical markers from which we classified the type of anemia and serum micronutrient levels. Diet was evaluated using a semiquantitative food frequency questionnaire. Results and conclusions: The most frequent type of anemia as normocytic normochromic, which can be associated to the patology per se. Patients with microcytic anemia showed a low iron consumption, as well as low iron serum levels. Overall, daily recommended allowances were not met; this can contribute to nutritional alterations.
\end{abstract}

Key words: cervical cancer, anemia, diet, iron, folic acid, vitamin $B_{12}$

Este trabajo fue recibido el 2 de Marzo de 2009 y aceptado para ser publicado el 15 de Noviembre de 2009.

\section{INTRODUCCIÓN}

La anemia se define como una disminución en la concentración sérica de hemoglobina $(\mathrm{Hb})$ cuyo valor es menor al de referencia. Para la Organización Mundial de la Salud (OMS) esta reducción se refiere a un valor por debajo de $12 \mathrm{~g} / \mathrm{dL}$ en mujeres adultas. (1) Sin embargo, este valor debe ser adecuado a la altura sobre el nivel del mar puesto que a mayor altitud, se reduce la presión parcial de oxigeno, condicionando hipoxemia relativa y policitemia compensadora. Por este motivo, en la Cd. de México, se considera un valor de $\mathrm{Hb}<13.5 \mathrm{~g} / \mathrm{dL}$ como indicativo de anemia. (2)

La anemia se presenta frecuentemente en los pacientes con cáncer; se ha reportado que más del $30 \%$ de los pacientes con tumores malignos la han padecido. $(3,4)$ La anemia nutricional se refiere a aquélla que se origina debido un aporte inadecuado de los nutrimentos hematopoyéticos: hierro, ácido fólico y cianocobalamina. (5) De acuerdo a la deficiencia, se presentarán diferentes alteraciones en los hematíes: la deficiencia de hierro se evidencia por un menor volumen celular, mientras que la deficiencia de folatos o cianocobalamina provocarán un aumento en el mismo.

Existe además, un tipo de anemia conocido como anemia de la enfermedad crónica. A nivel mundial es la segunda causa de anemia -después de la ferropénica- y entre sus causas se encuentran infecciones, cáncer, enfermedades autoinmunes, endocrinológicas e inflamatorias del riñón, entre otras. Lo anterior ha hecho que se le denomine también anemia por inflamación. En México, la prevalencia de este tipo de anemia es de 20 a $24.5 \%$, muy similar a la de otros países en desarrollo (6). 
Por su parte, el cáncer cérvico uterino $(\mathrm{CaCu})$ ocupa el tercer lugar en mortalidad relacionada con las neoplasias malignas en la población mexicana en general; en la población femenina, es la segunda causa de muerte por neoplasias malignas (7).

Como se ha mencionado, la anemia es una alteración frecuente en el paciente oncológico. En pacientes con $\mathrm{CaCu}$, se ha encontrado presente en hasta $51 \%$ como anemia leve (Hb $11-13 \mathrm{mg} / \mathrm{dL}$ ) y $17 \%$ como anemia (Hb $<11 \mathrm{mg} / \mathrm{dL}$ ). (8) Adicionalmente, se ha correlacionado la presencia de anemia con el estadio o progresión de la enfermedad, encontrándose una relación directamente proporcional: en estadio I se encontró $25 \%$ y en estadio II, $33 \%$ de pacientes con anemia y cáncer (9).

Dada la frecuencia e importancia de ambas patologías ( $\mathrm{CaCu}$ y anemia) en nuestro país, el objetivo del estudio fue determinar la presencia y tipo de anemia (de acuerdo a la morfología del eritrocito) y relacionarla a las concentraciones séricas de micronutrimentos hematopoyéticos (hierro, ácido fólico y cianocobalamina) y su respectiva ingestión dietética. De acuerdo a los resultados, se podría implementar un tratamiento coadyuvante a manera de mejorar el estado crónico que conlleva la enfermedad, así como orientar a las pacientes en el tipo de nutrición así como el uso de suplementos necesarios, en caso de presentar síndrome anémico para una mejor tolerancia al tratamiento quimioterapéutico, radioterapéutico y quirúrgico.

\section{SUJETOS Y MÉTODOS}

Se realizó un estudio transversal -previa autorización por los comités de Investigación y Ética del Hospital General de México- en cual se estudió a 55 pacientes (muestreadas de manera consecutiva durante el periodo de enero a noviembre de 2008) con diagnóstico de $\mathrm{CaCu}$ que fueron atendidas en el servicio de Gineco-Oncología del Hospital General de México a las cuales se les determinó la presencia y el tipo de anemia. Se determinaron los valores de $\mathrm{Hb}$, hematocrito (Hto), volumen corpuscular medio (VCM), hemoglobina corpuscular media (HCM), distribución eritrocitaria (RDW), ferritina y transferrina; así como las concentraciones séricas de hierro, ácido fólico y vitamina $\mathrm{B}_{12}$ en una muestra sanguínea que fue tomada a la paciente una vez consentida su participación en el estudio. Como análisis estadístico descriptivo, se determinaron las proporciones de anemia clasificada de acuerdo al tamaño celular y, posteriormente, se determinaron las medias de los indicadores bioquímicos antes mencionados a fin de ser comparados contra valores de referencia.

La evaluación dietética se llevó a cabo mediante la aplicación de un cuestionario de frecuencia alimentaria semicuantitativo validada para población mexicana por el Instituto Mexicano del Seguro Social; a través de ésta se determinó el consumo diario de hierro, vitamina $\mathrm{C}$, vitamina $A$, vitamina $B_{12}$, ácido fólico y calcio, para ser comparado con la referencia de ingesta recomendada.

Los criterios de inclusión fueron: pacientes mayores de 18 años con diagnóstico de $\mathrm{CaCu}$ estadios II y III sin sangrado transvaginal presente ni tratamiento oncológico previo que aún no iniciaran tratamiento para la patología en curso y que consintieran su participación en el estudio. Se excluyó a pacientes con transfusión sanguínea reciente.

\section{RESULTADOS}

La totalidad de las pacientes presentaron concentraciones de $\mathrm{Hb}$ menores $13.5 \mathrm{~g} / \mathrm{dL}$. En la Gráfica 1 se describe la clasificación de las pacientes de acuerdo a

\section{GRÁFICA 1}

Frecuencia del tipo de anemia en $\mathrm{CaCu}$

口Microcitica Hipocromica \% a Normocitica Normocromica \%

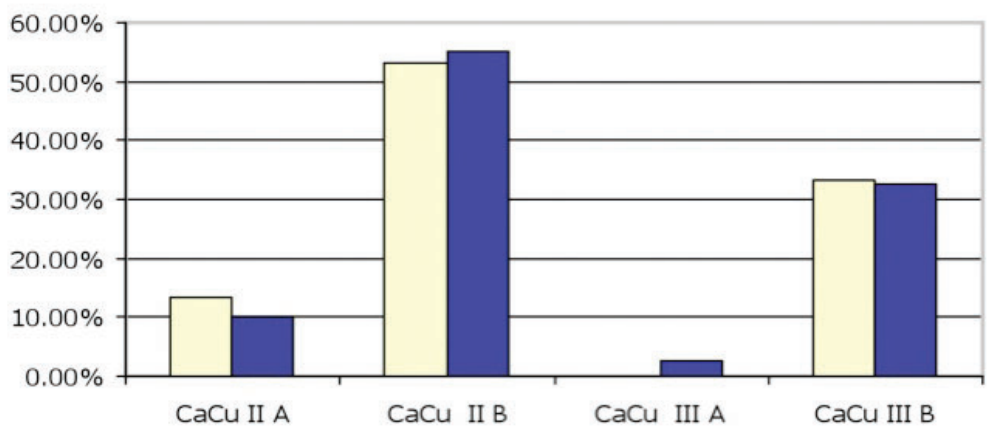


la morfología del eritrocito en anemia normocítica normocrómica o microcítica hipocrómica (ya que ninguna paciente presentó morfología macrocítica. La tabla 1 presenta la estadística descriptiva de la biometría hemática de las pacientes, una vez clasificadas de acuerdo al tipo de anemia presente. En la tabla 2 se presentan los resultados referentes a las concentraciones de los micronutrimentos hematopoyéticos. Finalmente, en la tabla 3 se describe el consumo de los micronutrimentos de interés. En este apartado, se encontraron valores muy diversos (desviación estándar amplia) que dan un intervalo donde el valor menor corresponde a una muy baja ingestión de hierro, misma que puede relacionarse a la concentración disminuida del mismo. Sin embargo, las pacientes con anemia por deficiencia de hierro sólo $27 \%(\mathrm{n}=15)$ presentan niveles por debajo lo normal de hierro. El hierro obtuvo una media de $30.08 \mathrm{mg}$ semanales en las mujeres con anemia microcítica y esto quiere decir que las pacientes cifra muy por debajo del valor normal (18 mg/día): en promedio ingieren 6.82 $\mathrm{mg}$ al día. Ya que la vitamina $\mathrm{C}$ influye en la biodis- ponibilidad del hierro, es muy importante incluirla en la dieta; el consumo semanal de esta vitamina fue de $418.05+/-229.54$, esto resulta en un consumo diario de $59.7 \mathrm{mg}$; al compararlo con el requerimiento diario nos damos cuenta que la media está bajo de lo normal (75 mg), pero en esta ocasión al sumar la desviación estándar el requerimiento diario sí se alcanza. Por su parte, el consumo de vitamina $\mathrm{B}_{12}$ resultó de $7 \mathrm{mg}$ al día, valor arriba de lo recomendado. Así mismo los resultados muestran que, de ácido fólico, las pacientes sólo consumen $57 \mathrm{mg}$ diarios de los $400 \mathrm{mg}$ recomendados. Por último observamos el valor del calcio, en donde de los $1000 \mathrm{mg}$ requeridos, solamente se consumen 162 $\mathrm{mg}$ al día. Este micronutrimento se analizó puesto que disminuye la absorción adecuada de hierro; sin embargo, ya que la ingesta es muy baja de ambos nutrimentos no se afectan de ninguna manera.

\section{DISCUSIÓN}

La anemia normocitica normocromica resulto ser la más frecuente en $73 \%$ de los casos. La causa de más

\section{TABLA 1}

\section{Características hematológicas de la muestra estudiada}

Indicador bioquímico (Valor normal)

Hemoglobina

(13.5-15 g/dL)

Hematocrito

$(38-46 \%)$

$\mathrm{VCM}$

(80-100 fL)

HCM

(27-31g/dL)

CHMC

(32-36 g/dL)

RDW

(10-14\%)

Eritrocitos

(4-5.310\% $/ \mathrm{ul})$

Reticulocitos

(0.5-2\%)

Albúmina

(3.5-5.5g/dL)
Tipo de Anemia

Microcitica Hipocromica

Media +/- DS

Normocitica Normocromica Media +/- DS

$9.86+/-1.68$

$11.31+/-2.37$

$34.13+/-5.86$

$89.42+/-6.68$

$71.6+/-5.69$

$29.73+/-1.97$

$26.12+/-14.84$

$33.87+/-1.59$

$30.98+/-2.34$

$14.89+/-2.46$

$18.58+/-4.22$

$3.79+/-1.50$

$2.02+/-1.12$

$1.70+/-1.36$

$3.3+/-0.26$

$3.14+/-0.63$ 
probable de este tipo de anemia es la existencia de la enfermedad crónica. Las pacientes con anemia microcítica hipocrómica, presentaron $\square$ en $60 \%$ - niveles de hierro sérico por debajo del valor normal; la transferrina, $24 \%$ resultó con niveles debajo de lo normal; en ferritina, $35 \%$ resultó por debajo de lo normal; por tanto se evidencia que, efectivamente, es por deficiencia de hierro que se presenta la anemia. Aunado a esto, es necesario recordar que las pacientes provienen de un nivel socioeconómico muy bajo, un motivo determinante para su estado nutricional. Ya que incluso en niveles socioeconómicos más elevados se observa este problema, era de esperarse esta situación con las pacientes, pues al momento de realizar las encuestas referían que la mayoría de las veces la obtención de los alimentos era difícil: por la situación de no contar con el dinero suficiente para obtenerlos o por la simple razón de vivir en las afueras de la ciudad y no tener acceso a ellos. Los alimentos que contienen hierro no están al alcance de estas personas como son las carnes rojas, huevos, leguminosas, cereales y vegetales verdes; además, para que el hierro de estos alimentos se pueda absorber de manera adecuada, también es necesario la presencia de las vitaminas, como la $\mathrm{C}$ y la $\mathrm{A}$; presentes en alimentos como frutas cítricas, vegetales verdes y el trigo (10).

Las 55 pacientes que se estudiaron se encontraban

TABLA 2

\section{Micronutrimentos a nivel sérico}

Indicador bioquímico

(Valor normal)
Tipo de anemia

Microcitica hipocromica Media +/- DS

Normocitica normocromica Media +/- DS
Hierro

(28-170 mg)

Ferritina

(12-150 mg/dL)

Transferrina

(192-382 ng/dL)

Acido Folico

(5-25 ng/dL)

Vitamina $\mathrm{B}_{12}$

(150-285 pg/mL)
$49.73+/-9.20$

$49.73+/-51.44$

$69.52+/-78.90$

$254.38+/-87.51$

$11.66+/-4.81$

$383.33+/-226.52$
$89.92+/-171.41$

$262.64+/-83.38$

$10.13+/-4.77$

$558.68+/-307.16$

TABLA 3

Características del consumo de nutrientes

\section{Indicador dietético}

(Valor normal)

\section{Microcitica hipocromica \\ Media +/- DS}

Tipo de anemia

Normocitica normocromica Media +/- DS

$39.92+/-13.65$

$50.14+/-11.93$

$857.57+/-44.73$

$2.28+/-42.24$

$57.57+/-11.11$

$1200.86+/-50.26$ 
en estadios en los cuales ya hay invasión más allá del útero e incluso la pared pélvica, en donde los sangrados transvaginales es la causa por la que las pacientes acuden a consulta, sin embargo en el momento de la toma de la muestra las pacientes no presentaban esta complicación; pero al sumarse los sangrados previos y la mala alimentación corroboramos que las pacientes necesariamente requieren una suplementación inmediata para prevenir estados más avanzados de anemia y una mejor respuesta al tratamiento, por supuesto que la alimentación tiene una importancia relevante, recomendando una dieta adecuada y suplementando con los nutrimentos necesarios, la calidad de la respuesta del organismo mejoraría indudablemente.

La diferencia entre anemias ferropénicas y no ferropénicas donde las variables hematológicas que resultaron con una $\mathrm{p}<0.05$ fueron el VCM y RDW, marcadores bioquímicos con los cuales se ha demostrado que se pueden clasificar las anemias por lo tanto con estos datos podemos confirmar lo que se menciona en la literatura que el VCM en pacientes con anemia ferropénica se encuentra disminuido y el RDW aumentado (11).

Finalmente, con este estudio hemos demostrado la necesidad de realizar intervenciones inmediatas en cuanto a necesidades alimenticias de hierro y suplemento vitamínico. Se demostró que no hay un consumo adecuado de nutrimentos y esto probablemente no hace posible una adecuada respuesta al tratamiento. También es necesario elaborar programas de nutrición para este tipo de pacientes ya que necesitan una guía de cómo nutrirse adecuadamente y con alimentos que contengan alto contenido de hierro, vitamínico y que esté al alcance de su economía ya que se observó que el nivel socioeconómico es la limitante que más sobresalto al momento de la encuesta.

\section{RESUMEN}

Introducción: La anemia es un síndrome que los pacientes con cáncer presentan frecuentemente, repercutiendo este fenómeno en su calidad de vida. En el cáncer cérvicouterino $(\mathrm{CaCu})$, la anemia se desarrolla en la mayoría de las pacientes sin saber la causa que la produce; como el sangrado que se presenta en estadios avanzados o por el tipo de dieta baja en hierro y otros micronutrimentos. Objetivo: Determinar el tipo de anemia, las concentraciones séricas de hierro sérico, ferritina, transferrina, ácido fólico y vitamina $\mathrm{B}_{12} \mathrm{y}$ relacionarlo con la ingestión dietética. Sujetos y métodos: Se realizó un estudio transversal, con 55 pacientes con diagnóstico de cáncer cérvico uterino sin sangrado transvaginal presente ni tratamiento oncológico. Se tomó una muestra de sangre a fin de determinar pará- metros bioquímicos a partir de los cuales se determinó la presencia de anemia y el tipo de ella, así como concentraciones de micronutrimentos hematopoyéticos. Se aplicó una frecuencia alimentaria semicuantitativa para evaluar la ingestión dietética. Resultados y conclusiones: La anemia de mayor frecuencia fue de tipo normocítica normocrómica, misma que puede asociarse a la patología en curso. Las pacientes con anemia microcítica mostraron concentraciones bajas de hierro sérico, así como un bajo consumo del mismo. En general, no se cubrieron las recomendaciones de ingestión de micronutrimentos, aspecto que puede favorecer las alteraciones nutricionales.

Palabras clave: cáncer cervico uterino, anemia, consumo dietético, hierro, ácido fólico, vitamina $\mathrm{B}_{12}$

Dirigir la correspondencia a:

Profesora

Vanessa Fuchs

Hospital General de México

Servicio de Oncología. Unidad 111

Dr. Balmis \#148, Col. Doctores. Del.

Cuauhtémoc C.P. 06726

México, D.F.

Tels. (5255) 27892000 ext. 1062, 1510

Correo electrónico: fuchsvanessa@yahoo.com

\section{BIBLIOGRAFÍA}

1. Bayo E, Muñoz L, Sánchez A, Alonso E, Ortiz M, Romeo J. et al. Incidencia y prevalencia de anemia en pacientes que reciben radioterapia. Oncología (Barc.) 2004; 27(9): 19-26.

2. Terrés S, Arturo M, Razo D, Formula Roja: Límites de referencia biocronológicos y niveles de decisión clínica en población mexicana. Rev Med IMSS 2000; 38(4): 313-21.

3. Jaime-Caro J, Salas M, Ward A, Goss G. Anemia as an independent prognostic factor for survival in patients with cancer. Cancer 2001; 91(12): 2214-21.

4. Obermair A, Handisurya A, Kaider A, Sevelda P, Kolbl H,Gitsch G. The relationship of pretreatment serum hemoglobin level to the survival of epithelial ovarian carcinoma patients. Cancer 1998; 83(4):726-31.

5. Reboso-Perez J. Indicadores bioquímicos de la deficiencia de Hierro. Rev Cubana Aliment Nutr 1997; 11(1): 64-7.

6. Morales-Adrián J, Martínez-Magaña J. Anemia de la enfermedad crónica. Revista de Hematología 2006; 7(3): 99-107.

7. Estadísticas de mortalidad en México: muertes 
registradas en el año 2001. Sal Pub Mex 2002; 44(6): 565-81.

8. Dunst J, Kuhnt T, Strauss H, Krause U. Anemia in cervical cancers: impact on survival, patterns of relapse, and association with hypoxia and angiogenesis. Int J Radiat Oncol Biol Phys 2003; 56(3): 778-87.

9. Marchal C, Rangeard L. Anemia impact on treatments of cervical carcinomas. Cancer Radiother
2005; 9(2): 87-95.

10. Pérez-Lizaur AB, Casanueva E, Kaufer-Horwitz M; Arroyo P, editores. Nutriología médica. $2^{\mathrm{a}}$ ed. México: Ed. Médica Panamericana; 2001. pp. 449-58.

11. Romero J, Carbia C, Ceballo F, Díaz N, Índice de distribución de glóbulos rojos (RDW): su aplicación en la caracterización de anemias microcíticas e hipocrómicas. Medicina 1999; 59: 17-22. 